
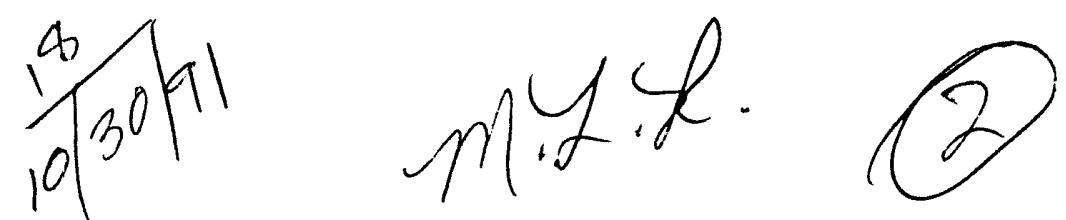

\title{
Robotic Capabilities for Printed Wiring Assembly Processing
}

\author{
K. J. Groot \\ KCP-613-4420
}

Published October 1991

Final Report

Prepared for the United States Department of Energy Under Contract Number DE-ACO4-76-DP00613. 
This report was prepared as an account of work sponsored by an agency of the United States Government. Neither the United States Government nor any agency thereof, nor any of their employees, makes any warranty, express or implied, or assumes any legal liability or responsibility for the accuracy, completeness, or usefulness of any information, apparatus, product, or process disclosed, or represents that its use would not infringe privately owned rights. Reference herein to any specific commercial product, process, or service by trade name, trademark, manufacturer, or otherwise, does not necessarily constitute or imply its endorsement, recommendation, or favoring by the United States Government or any agency thereof. The views and opinions of authors expressed herein do not necessarily state or reflect those of the United States Government or any agency thereof.

Printed in the United States of America.

This report has been reproduced from the best available copy.

Available to DOE and DOE contractors from the Office of Scientific and Technical Information, P. O. Box 62, Oak Ridge, Tennessee 37831; prices available from (615) 576-8401, FTS 626-8401.

Available to the public from the National Technical Information Service, U. S. Department of Commerce, 5285 Port Royal Rd., Springfield, Virginia 22161. 
$\mathrm{KCP}-613-4420$

Distribution Category UC-706

$$
\begin{aligned}
& \text { KCP }-613-4420 \\
& \text { DE92 } 002083
\end{aligned}
$$

ROBOTIC CAPABILITIES FOR PRINTED

WIRING ASSEMBLY PROCESSING

K. J. Groot

Published October 1991

Final Report

K. J. Groot, Project Leader

Project Team:

J. R. Dokos 
ROBOTIC CAPABILITIES FOR PRINTED WIRING ASSEMBLY PROCESSING

KCP-613-4420, Final Report, Published October 1991

Prepared by K. J. Groot

An investigation was conducted into the potential use of robotics in the fabrication processes for printed wiring assemblies. Robotic workcelis to tin component leads, freform component leads preparatory to insertion into printed wiring boards (PWBS), bin components in proper sequence for manual insertion operations, and automatically insert components into PWBs were developed and fabricated. Additional capabilities were developed for the tinning workcell that had been released in 1985. An insertion workcell is anticipated to be released to production in the near future.

$B B: 1 j s$

TR3/ab 


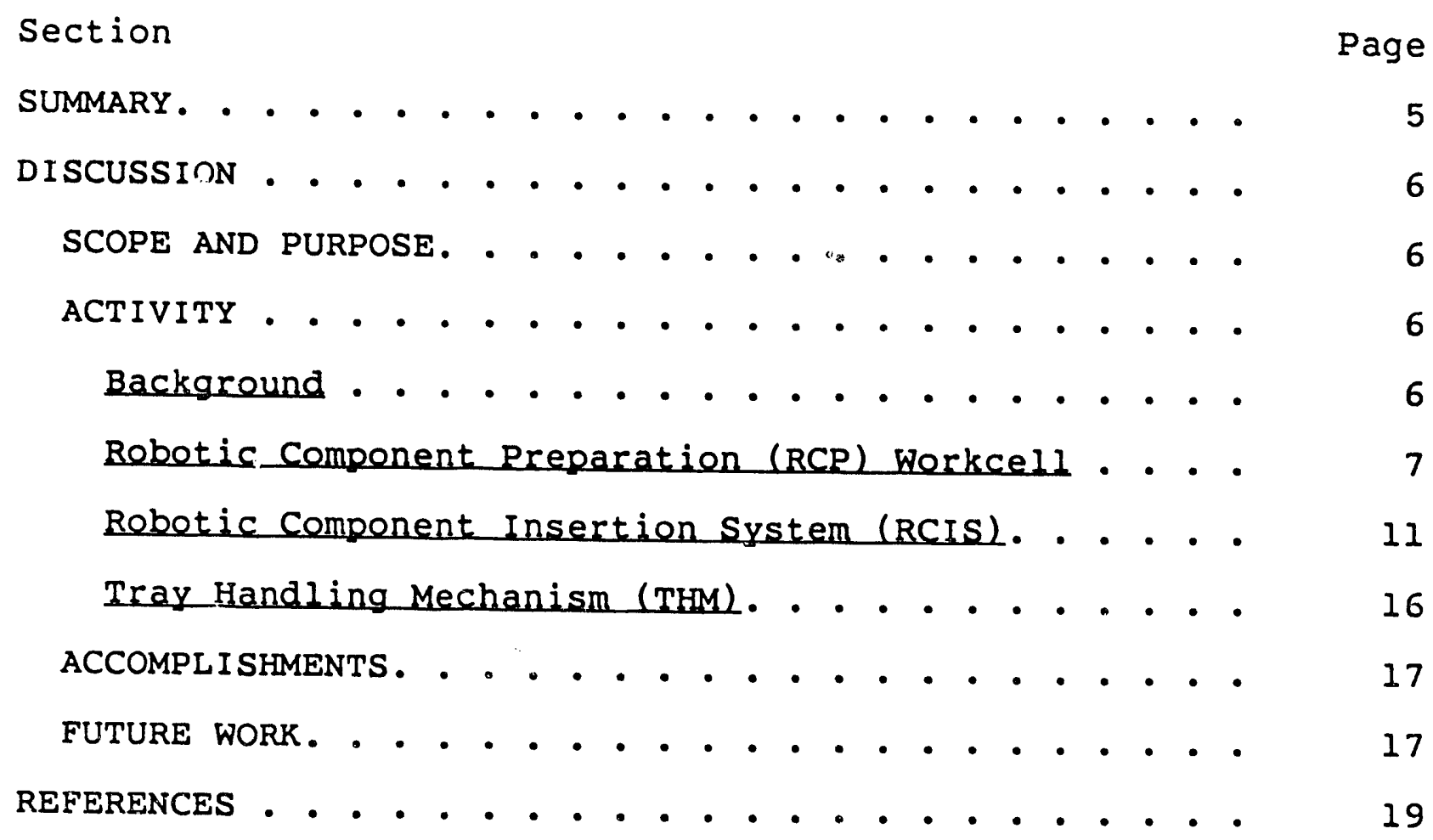




\section{ILLUSTRAT IONS}

Figure

1

2

3

4

5

6

7

8

9

10

Robotic Component Preparation (RCP) Workcell (P-129495) . . . . . . . . . . . . 8

Workcell With RCP Robot (P-129482) . . . . . 9

RCP Robot (P-129489) . . . . . . . . . 10

RCP Component Lead Cutoff and Preform Mechanism (P-137373) . . . . . . . . . . . . 11

Preformer With Stepper Motors (PH-144003). . . 12

Marking orientation Fixture (R09029-01). . . 13

Vibratory Feeder for Radial-Lead and Transistor Components $(\mathrm{P}-137375)$. . . . . 14

SCARA-Arm Robot (PH-143998). . . . . . . 15

Robotic Component Insertion System

(PH-143997)................. 16

Tray Handling Mechanism (PH-144001). . . . . 18 
Automation is widely used in the electronic industry for the manufacture of printed wiring assemblies (PWAs). Literature reviews and visits with some automation vendors revealed that robotics are used. when processing flexibility is required.

Robotic development work at the Allied-Signal Inc., Kansas City Division (KCD) resulted in the fabrication of three robot workcelis. The capabilities of two robotic Component preparation (RCP) workcells, that had been developed and fabricated to tin component leads, were increased by development on these processes. A Robotic Component Insertion System (RCIS) was developed and fabricated to insert components directly into a printed wiring board (PWB).

The RCP workcells were developed to use robotics to perform some of the manual processes on PWA components. These robotic processes prepared the components for the subsequent insertion and soldering in the PWAs. The first workcell was released for production use in 1985. The RCPs have proven to be reliable with resultant cost savings of up to $\$ 50,000 /$ year. Development work increased the capabilities of these RCPs and increased the cost savings by $\$ 30,000$ per year.

The RCIS workcell design was developed to automatically insert components into PWBs. A specification to meet this design was written, an order placed, and the RCIS fabricated and installed at KCD. The system is anticipated to be released for production use in the near future. 
DISCUSSION

\section{SCOPE AND PURPOSE}

Development work was performed to evaluate automated processes that were economically feasible to be used for printed wiring assembly (PWA) processes. Robotics was chosen to provide the processing flexibility that was not available with other automation processes. Robots were developed to replace time consuming, tedious, and hazardous manual operations currently used in KCD PWA manufacturing. Three robotic workcelis were developed, fabricated, and installed.

\section{ACTIVITY}

\section{Background}

An extensive evaluation of automation used in the electronic assembly industry revealed that hard automation is used primarily for processes involving large volumes of a single design. This type of automation does not operate satisfactorily with the KCD manufacturing scheme that involves many designs with low volumes. An extensive review of trade shows, seminars, and magazines revealed that robotics could provide the processing flexibility required by $\mathrm{KCD}$.

Initial robotic development efforts resulted in the fabrication of the Robotic Component Preparation (RCP) workcell. The RCP incorporated a robot to replace the manual process of tinning component leads. Automation of the tinning process was selected to relieve the operator of extensive manual operations. The cost savings for this workcell was estimated at $\$ 50,000 /$ year. The RCP was released to production in FY85. In order to meet KCD PWA schedules, a second RCP was fabricated and released to producticin. Continued development work resulted in additional capabilitiss being added to the RCP that increased the cost savings potential to $\$ 80,000 /$ year.

Development work was performed to incorporate the use of robotics into the $P$ WA component insertion process. Initial contact with vendors revealed several companies were offering systems that could perform these assembly processes. The design requirements were developed, a specification written, and RFQs submitted to interested vendors resulting in a purchase order being placed in 1986. The system was shipped and installed at KCD in 1988. Release for production use is expected in the near future. 


\section{Robotic Component Preparation ( $R C P$ ) Workcel1}

Axial-leaded components represent nearly $75 \%$ of the components used in KCD PWAs. The leads on each of these components must be tinned dipped in solder to insure the solderability when inserted into a printed wiring board (PWB). The manual tinning process used at $K C D$ requires the operator to carefully hand-dip each lead of the components into a 500 to $600^{\circ} \mathrm{F}$ solder pot to a specific distance from the component body and for a specified time. The best operators do not consistently meet all of the requirements. Care must also be exercised by the operators to prevent injury to themselves or damage to the components. The RCP lead tinning workcell (Figures 1 and 2 ) was developed to control the amount of flux on axial-lead component leads, control the depth and length of time the leads are in the wave solder pot, spray clean the excess flux from the lead and body, and place the components in the correct pocket of the component locator trays. The RCP incorporated a robot (Figure 3 ) to automatically perform these tinning operations in a reliable and safe manner. The first $R C P$ was released to production in the lst Quarter of 1985 with an initial cost savings estimated at $\$ 50,000 /$ year. A second RCP was fabricated and released to production to meet the KCD component tinning schedule demands.

Development work on the RCP was continued resulting in additional processes being developed and added to the workcell capability. ${ }^{1}$ These processes included the following.

Lead Trimming and Preforming Mechanisms

A lead trimming and preforming mechanism (Figure 4 ) was added to the RCP to trim the leads to a $2.000 \mathrm{in.}$ length. This length was chosen to standardize the lead length for use with the RCP and RCIS component handling requirements. The RCP robot places the component into the mechanism and the lead is trimmed on command from the robot controller. This mechanism can also preform axial-lead component leads to $0.300,0.325$, or 0.375 in., depending on which die is installed in the mechanism. To further expand the capability of the RCP, a preformer was purchased and stepper motors attached (Figure 5). These motors enabled the preformer to automatically preform the leads to the proper lead spacing upon command from the robot controller. The preformer is capable of preforming leads between $0.275 \mathrm{in}$. to 1.500 in.

\section{Component Marking Orientation Fixture}

Except for color-banded resistors, axial-lead components have marking requirements on the component body to identify the component. The assembly process requires this marking to be facing up from the PWB after the components are inserted. 


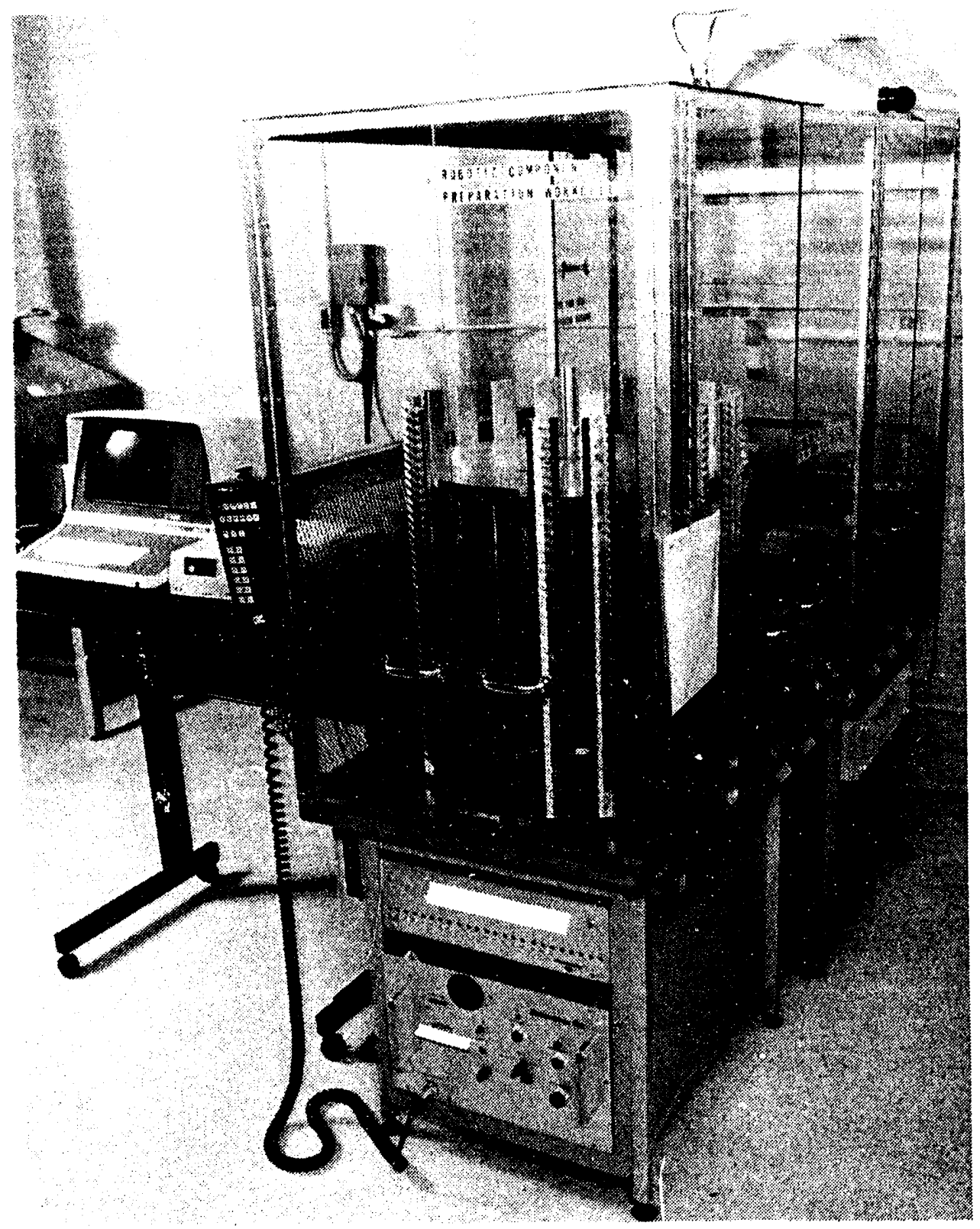

Figure 1. Robotic Component Preparation (RCP) Workcell

Inspection must verify the marking to ensure that the correct component has been inserted into the proper location. An initial look at vision systems to properly read and locate the marking were reviewed at KCD. These systems proved to be impractical based on the wide variety of body colors, body sizes, and marking fonts. Therefore, a process was developed to mechanically align this marking during the RCP preforming operations. The production operator is required to bend the last $0.250 \mathrm{in}$. of the lead at 90 degrees to the body, away from 


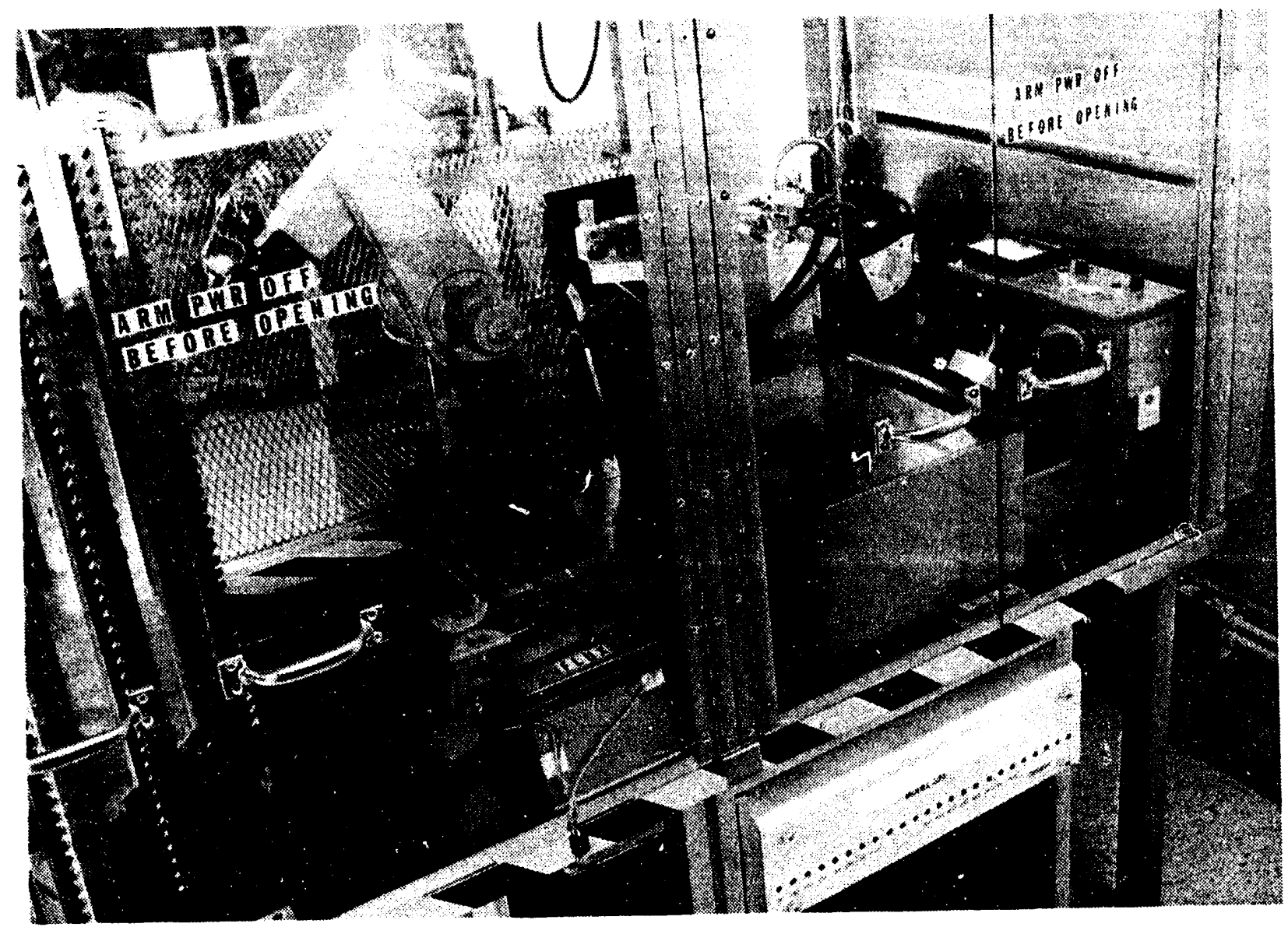

Figure 2. Workcell with RCP Robot

the marking. The component is then loaded into the RCP carousel. A fixture (Figure 6 ) was added to the R.CP adjacent to the trimming and preforming mechanism. The robot places the component into the horizontal slot in this fixture and opens the end-effector fingers. The robot then moves the component vertically and, just prior to emerging from the slot, re-grips the component body. The slots cause the component to rotate so that the bent leac always faces down when the component emerges from the fixture slot. This action ensures that the marking is in a known and consistent orientation in relation to the robot fingers. The robot can then place the component into the preforming mechanism and the leads will be preformed with the marking in the correct position.

The addition of these processes increased the estimated cost savings capability of the RCP by an additional $\$ 30,000 /$ year. The robots used on these workcells are no longer available from the manufacturer, which may create a probl"m in the future. Additional development may be needed to incorporate new robots to perform the RCP functions. 


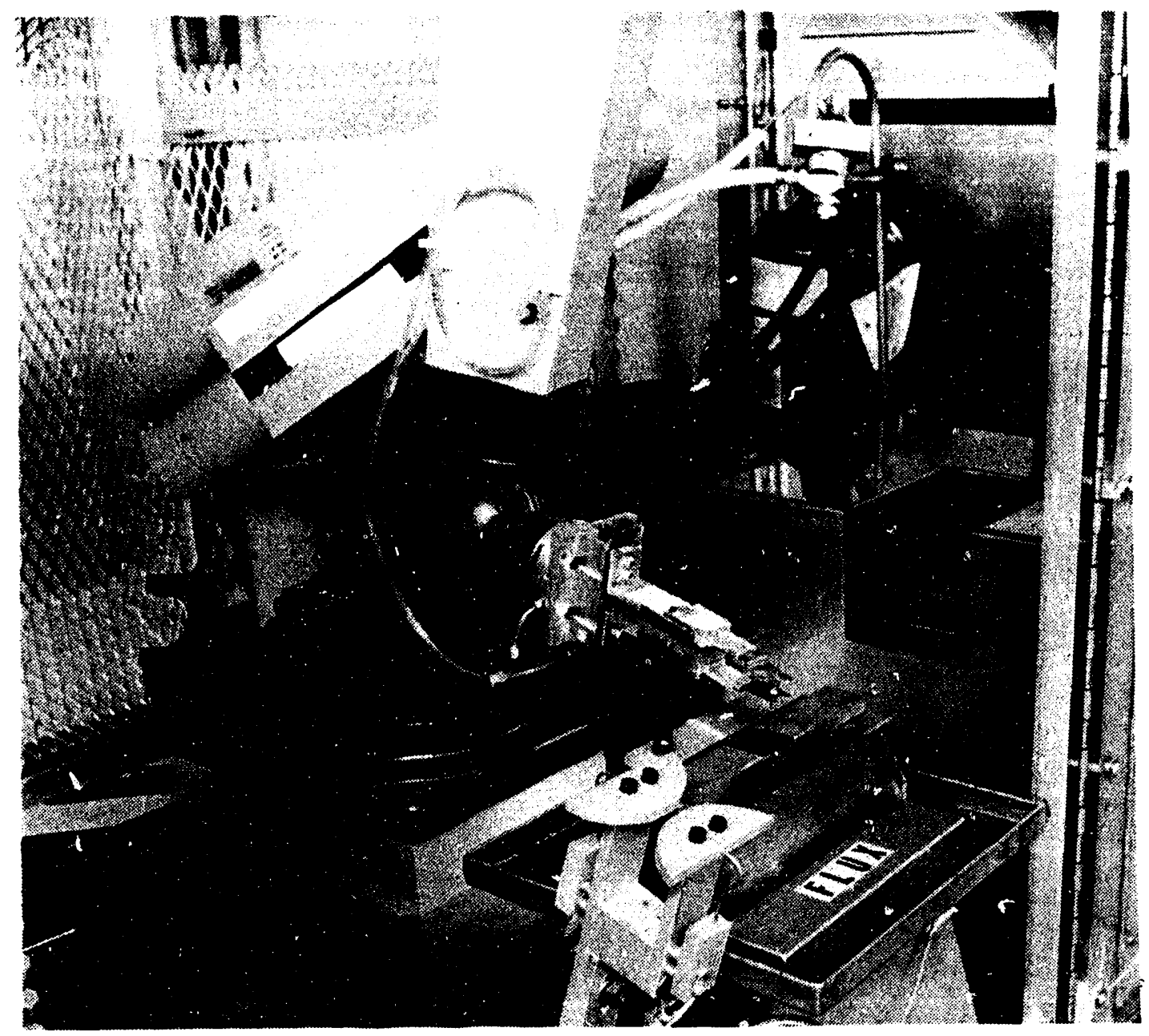

Figure 3. RCP Robot

Development work has also been performed to expand the tiniling capabilities in the RCPs to include radial-lead component tinning. A method was devised and incorporated on one cif the RCPs. Vibratory feeders were added to the RCP (Figure '7) to provide the capability to tin radial-lead components. These feeders are capable of handling radial-lead capacitors $(0.200$ and 0.300 in. body width) and transistors $(0.100$ and 0.200 in. diameter lead spacing). The vibratory action moves the components to the end of the track where the robot can pick them up. Upon command from the controller, the robot will change end-effector $f$ ingers in order to be able to pick up the correct components. This process requires at least three additional robot end-effector $f$ ingers to be able to pick up the radial-lead components. Because of the low quantity of these types of components and the limited resources on this development order, the required development work has not been completed to release this process to production. 


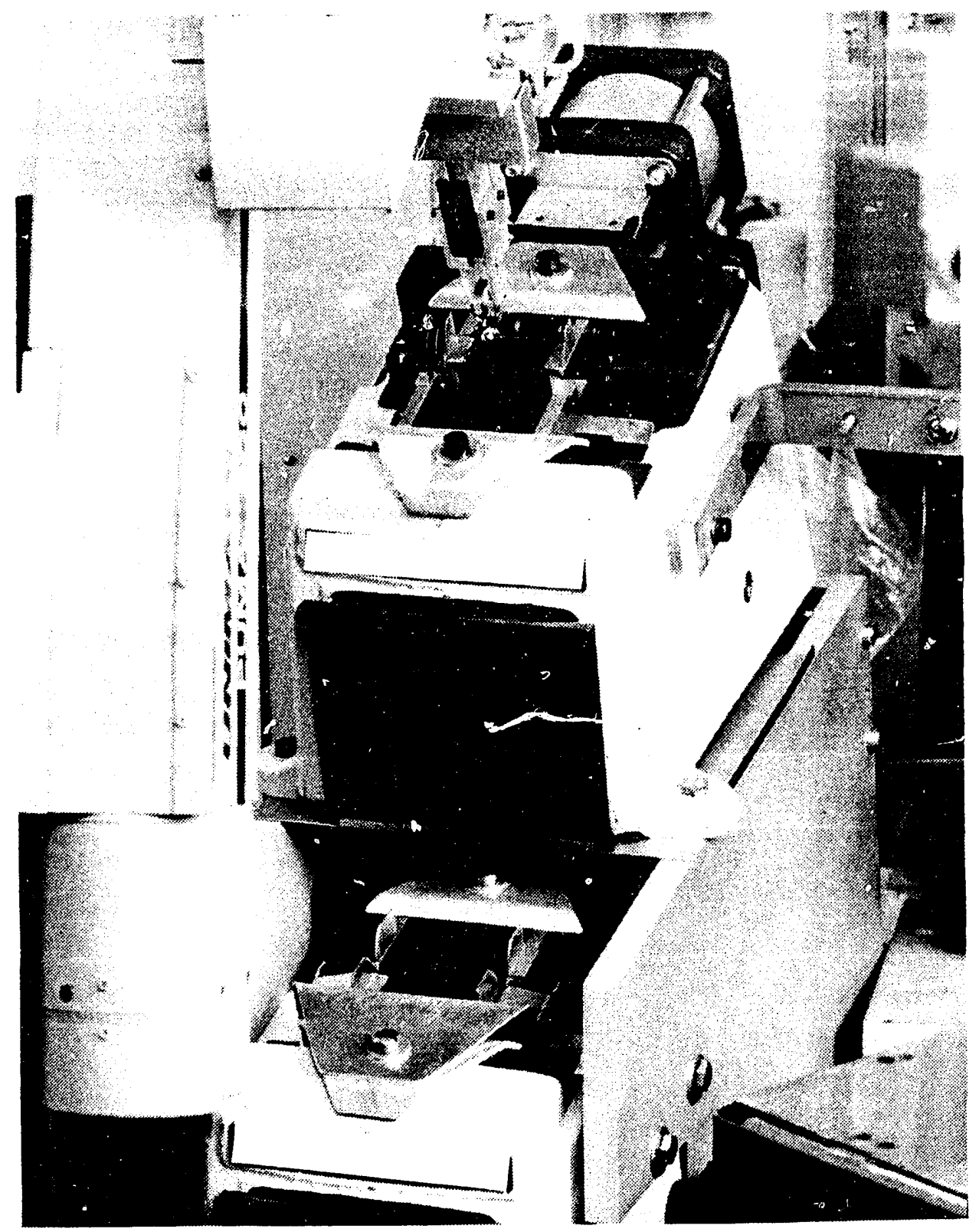

Figure 4. RCP Component Lead Cutoff and Preform Mechanism

Robotic Component Insertion system (RCIS)

The RCIS workcell design was developed after gathering information from various vendors and trade shows resulting in a specification being written to establish the system requirements. 2 These requirements were that the RCIS must be able to 


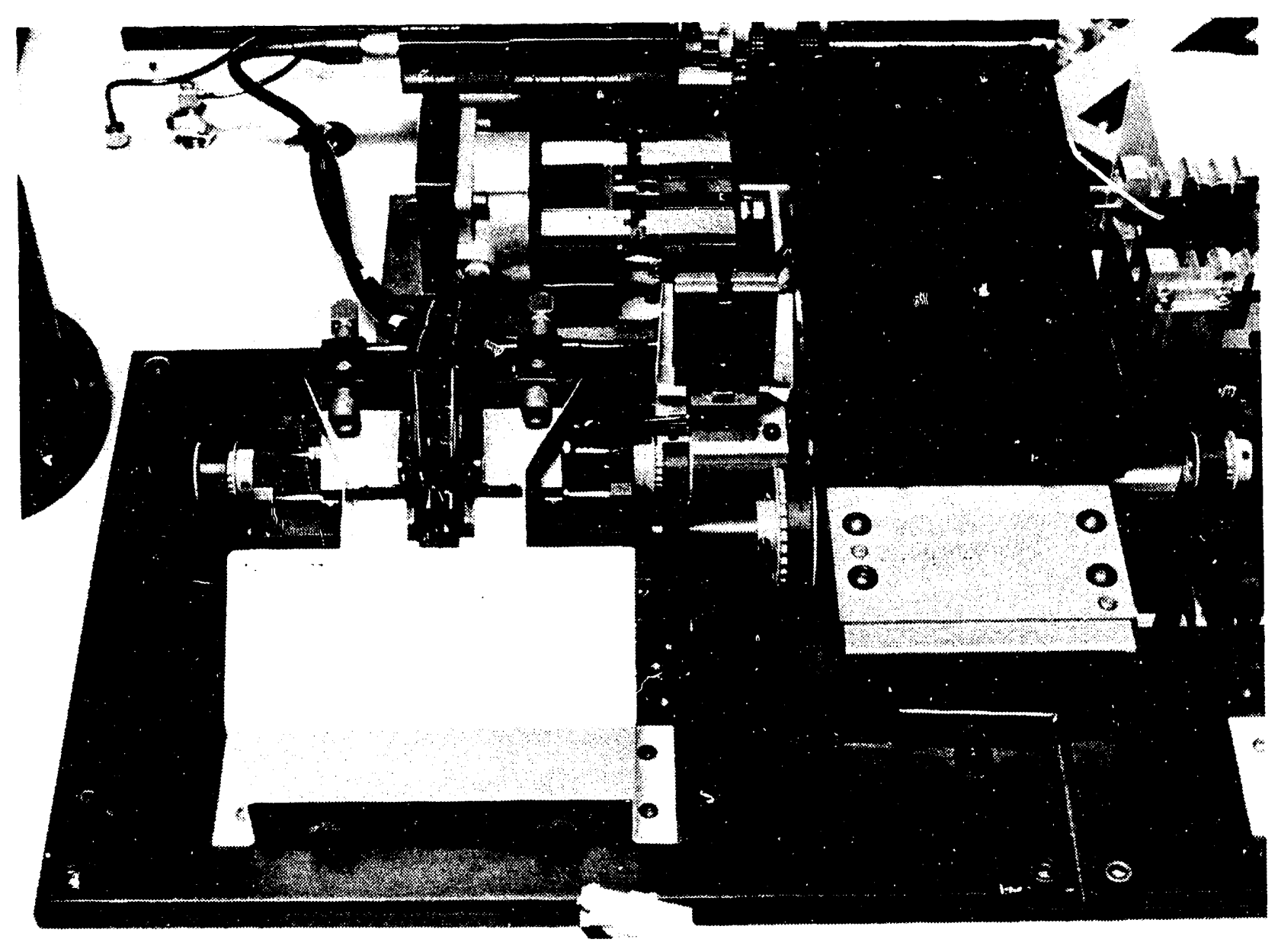

Figure 5. Preformer with Stepper Motors

- handle axial-leaded, radial-leaded, transistors, and Dual-inline-Planar (DIP) components;

- establish the location and orientation of the the PWB using a vision system to measure the location of the tooling holes and establish a reference frame based on the actual location measured;

- preform the component leads to the proper spacing concrolled by the system controller;

- electrically check the components and compare the measured value with data in the system database;

- measure the component body length and diameter using a vision system and compare the measured values with data in the system database; 


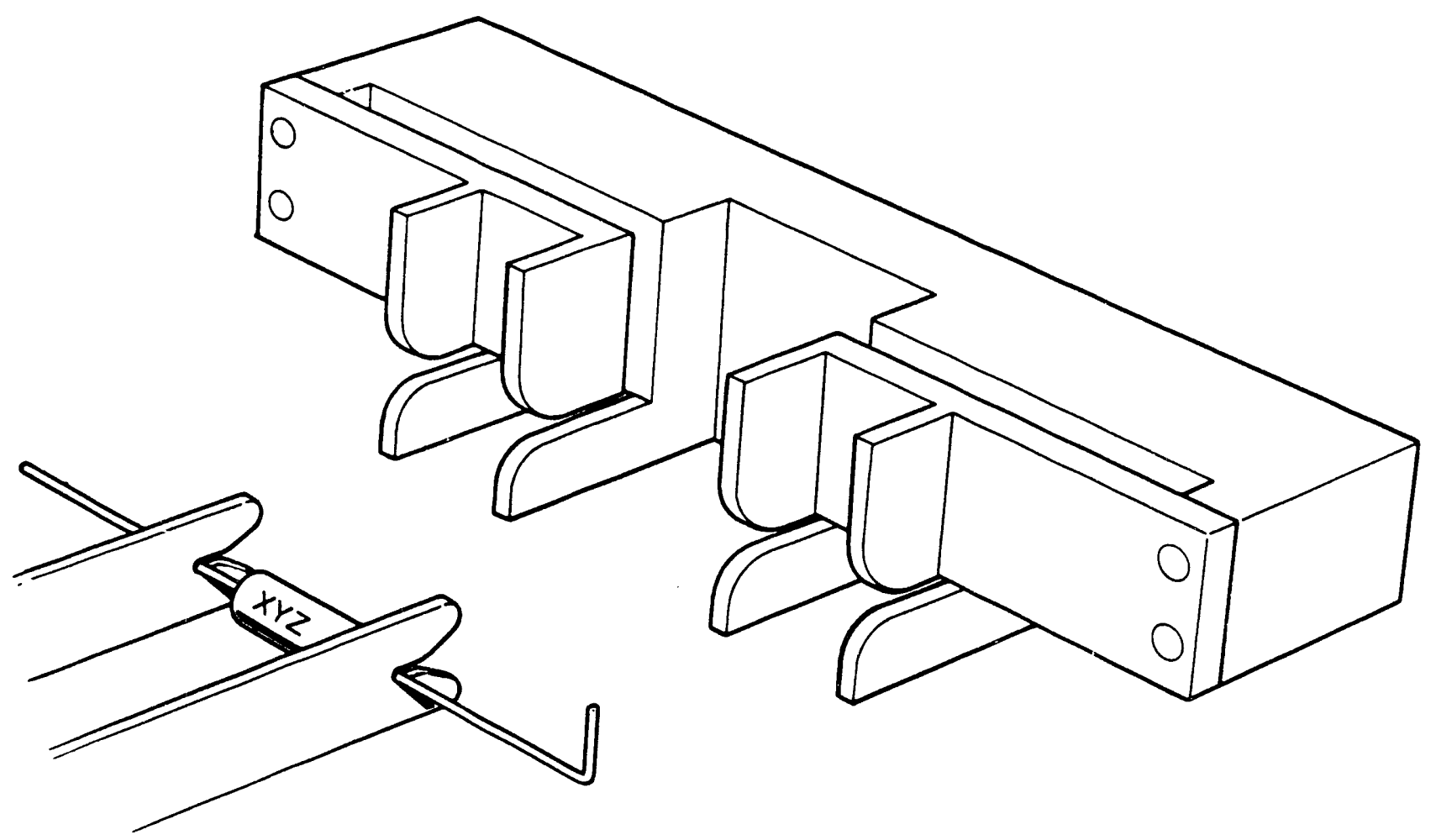

Figure 6. Marking Orientation Fixture

- verify the lead location using a vision system to establish a new reference frame based on the measured location of the lead in relation to the robot end-effector fingers;

- insert component leads into a PWB using a force sensing end-effector to verify when the component lead goes through the hole in the PWB;

- automatically perform a search routine if the lead does not go into the PWB hole on the first try; and

- clinch the component lead on the bottom side of the PWB to any angle in one degree increments.

The vendor chose to fabricate the RCIS selected a 4-axis SCARAarm robot (Figure 8 ) because of the capability and reliability factors which are required for this type of assembly process based on their experience building automation equipment using robotics. The vendor had fabricated other machines that could do most of the operations required of the RCIS, but had never built a machine that could do all these operations. The complexity of the RCIS was compounded by the 


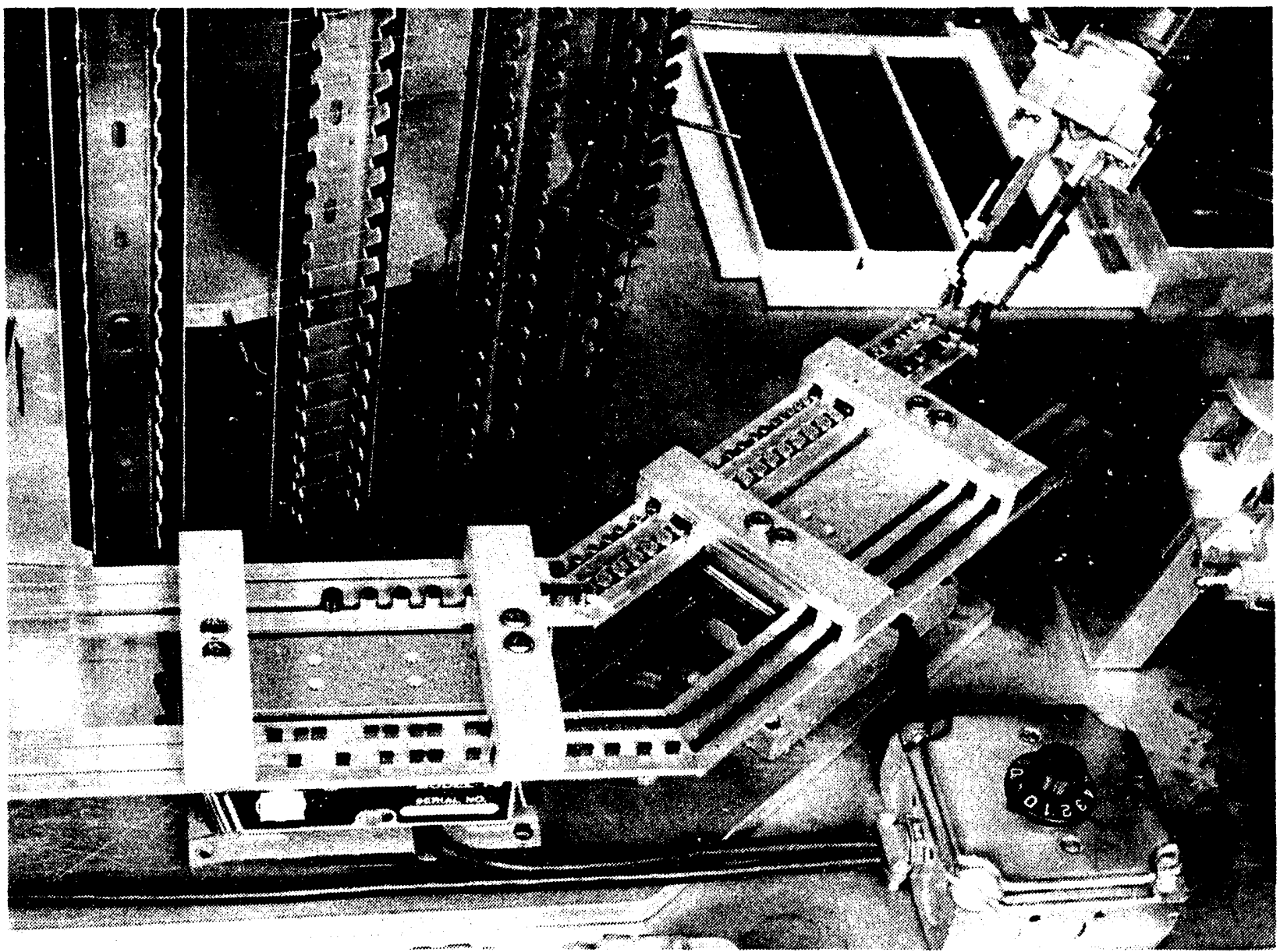

Figure 7. Vibratory Fe əder for Radial-Lead and Transistor Components

wide range of component types and body sizes, component lead diameters from 0.015 to 0.040 in. diameter, a wide range of lead preform dimensions, large differences in printed wiring board (PWB) material flexibility and thickness, and tight tolerances between the lead diameter and the PWB hole diameter. The mechanical portion of the RCIS was assembled within the time forecast, but the software took nine to twelve months longer than anticipated. Other delays occurred when interface design changes were required between the RCIS and the KCD processes. The RCIS was originally promised to be shipped to KCD in the second quarter of 1987, but was actually delivered and installed at KCD in the third quarter of 1988 (Figure 9).

Problems at KCD kept the RCIS system from being released for production use in 1989. These problems included

- jamming of the component presentation system causing some electrical switches to break which had to be replaced; 


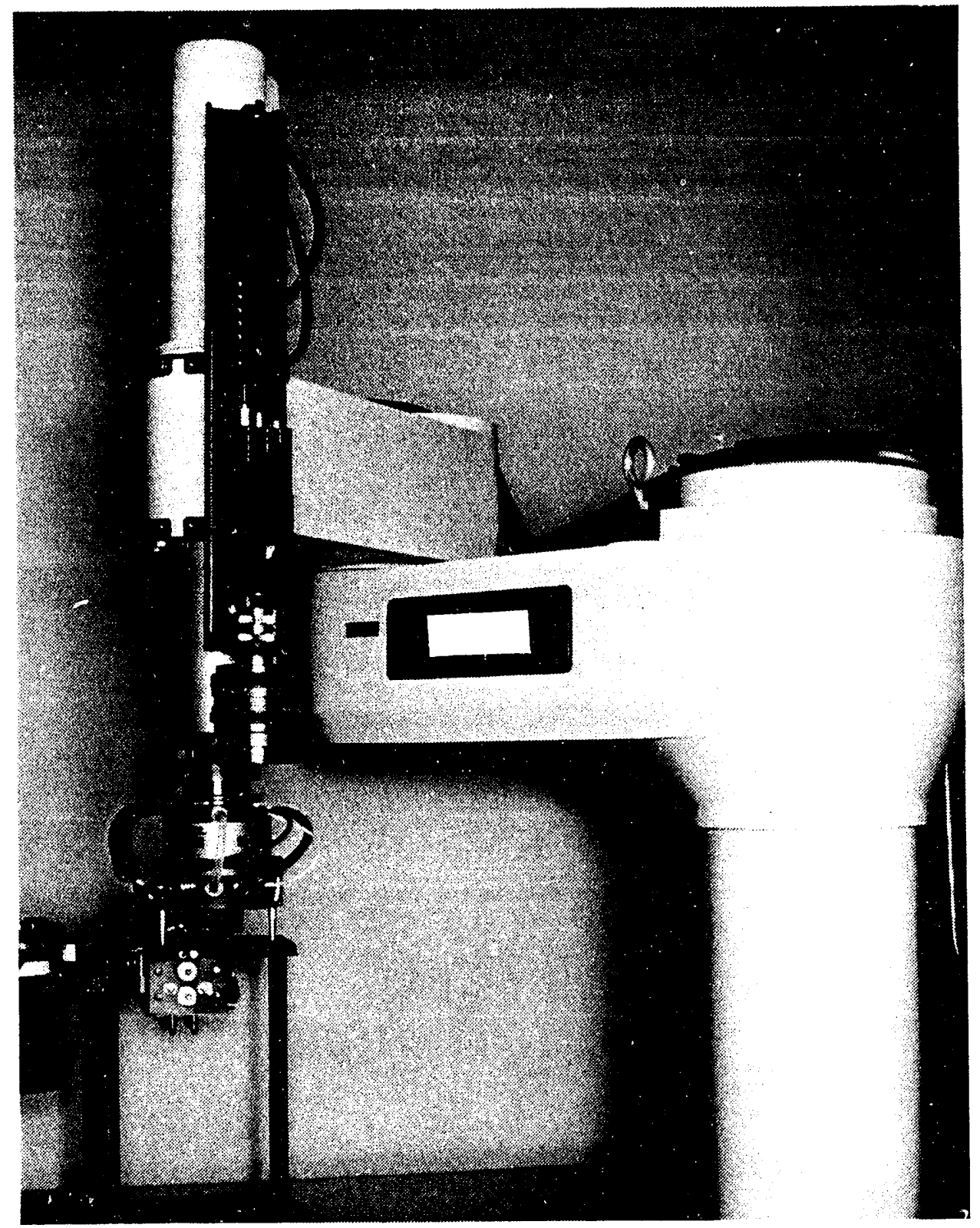

Figure 8. SCARA-Arm Robot

- delayed warranty work by the vendor while waiting to obtain the proper approval for their engineer to be admitted into $\mathrm{KCD}$;

- redesign and fabrication of axial-lead and radial-lead component trays to hold the components accurately enough for the robot to consistently pick up the components and to ensure that the leads did not bend during placement into the trays; and

- replacing of the parts handing tool controller motor. 


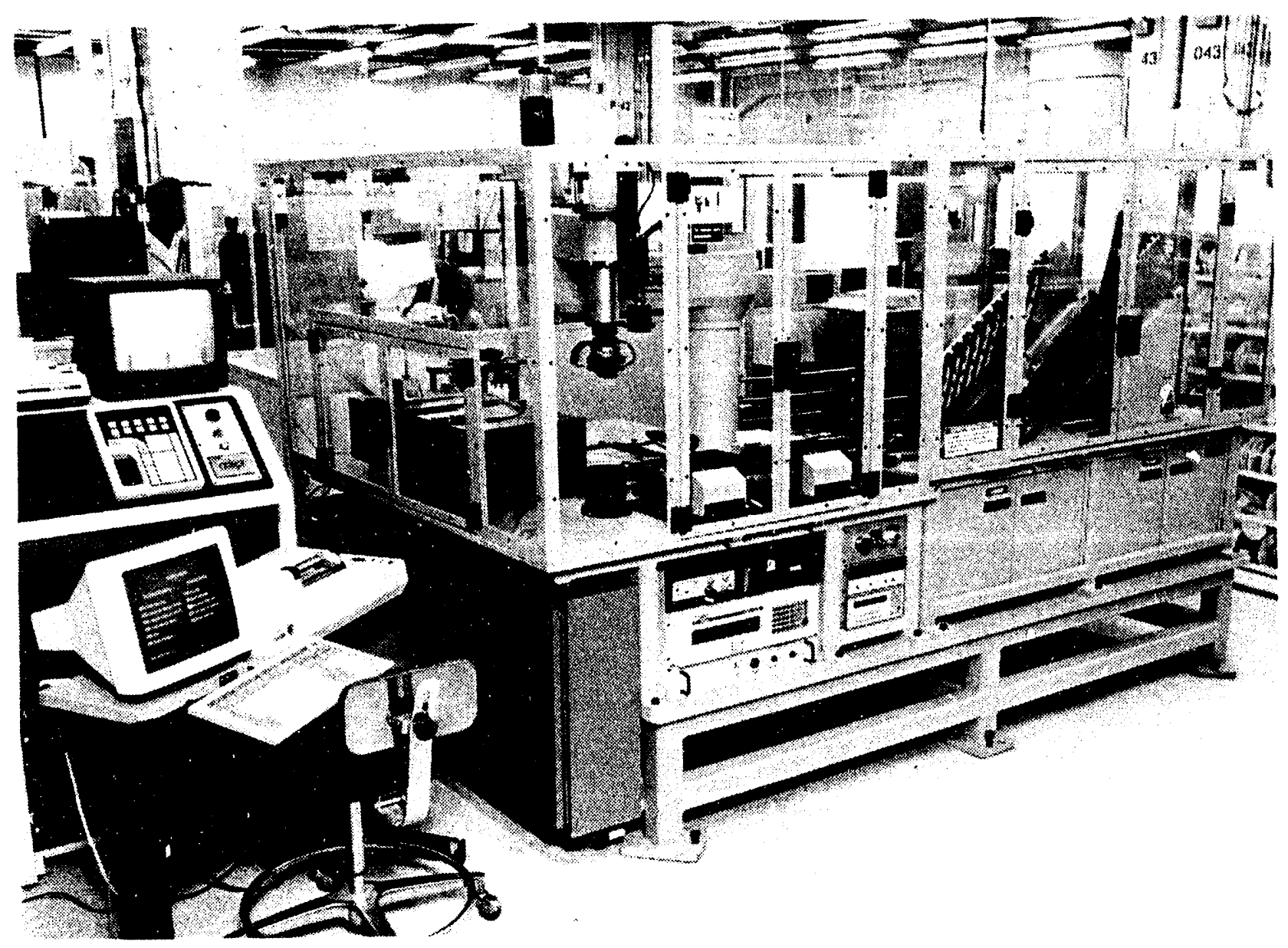

Figure 9. Robotic Component Insertion System

Development work on the RCIS at KCD is continuing to complete the development and release the RCIS for production use.

The original cost savings estimated for the RCIS was based on the RCIS being received from the vendor with ten PWAs preprogrammed into the controller. The system was programmed with these ten PWAs; however, delays in installing and releasing the RCIS to production have resulted in the loss of most of the original ten PWAs. This loss was caused when the processing responsibility for these PWAs was transferred to a different department and/or the scheduled build of the PWAs was completed. After the RCIS is released to production and in order to realize the originally estimated cost savings, additional PWAs must be programmed into the RCIS to replace the PWAs lost.

Tray Handing Mechanism (THM)

The RCP was designed to off-load the components into component locator trays. The RCIS component presentation system consists of 7 by 9 in. vacuum molded plastic trays which present the 
components to the robot. The THM specification was developed to interface between the RCP and the RCIS. This mechanism was specified to hold 36 of the RCIS plastic component trays, place the trays one at a time into the RCP, communicate with the RCP controlier to know when to change a full tray for an empty tray, and allow the easy removal of the full trays by the operator for loading into the RCIS. The RCIS vendor was also awarded the contract for the THM. They designed, fabricated, and shipped the THM to KCD in 1988 (Figure 10).

\section{ACCOMPLISHMENTS}

The PWA robotics development work has resulted in two Robotic Component Preparation (RCP) workcells, a Tray Handling Mechanism (THM), and the Robotic Component Insertion system (RCIS). The RCPs have been released for production use since 1985 resulting in cost savings up to $\$ 80,000 /$ year. The THM and RCIS were fabricated and installed at KCD and are expected to be released in the near future. The use of robotics has reduced the quantity of standard hours required to build PWAs, improved the reliability of the component processing, and will provide greater cost savings as additional workcells are released to production.

\section{FUTURE WORK}

A review of the RCPs will be made periodically to determine if development work will need to be started toward replacing the present robots. The RCIS will be analyzed for cost savings after the workcell is released to production. No new robotic development work is anticipated at this time, but KCD will continue to monitor the trade shows, vendors, and trade magazines for any new technology that may improve the PWA manufacturing. 


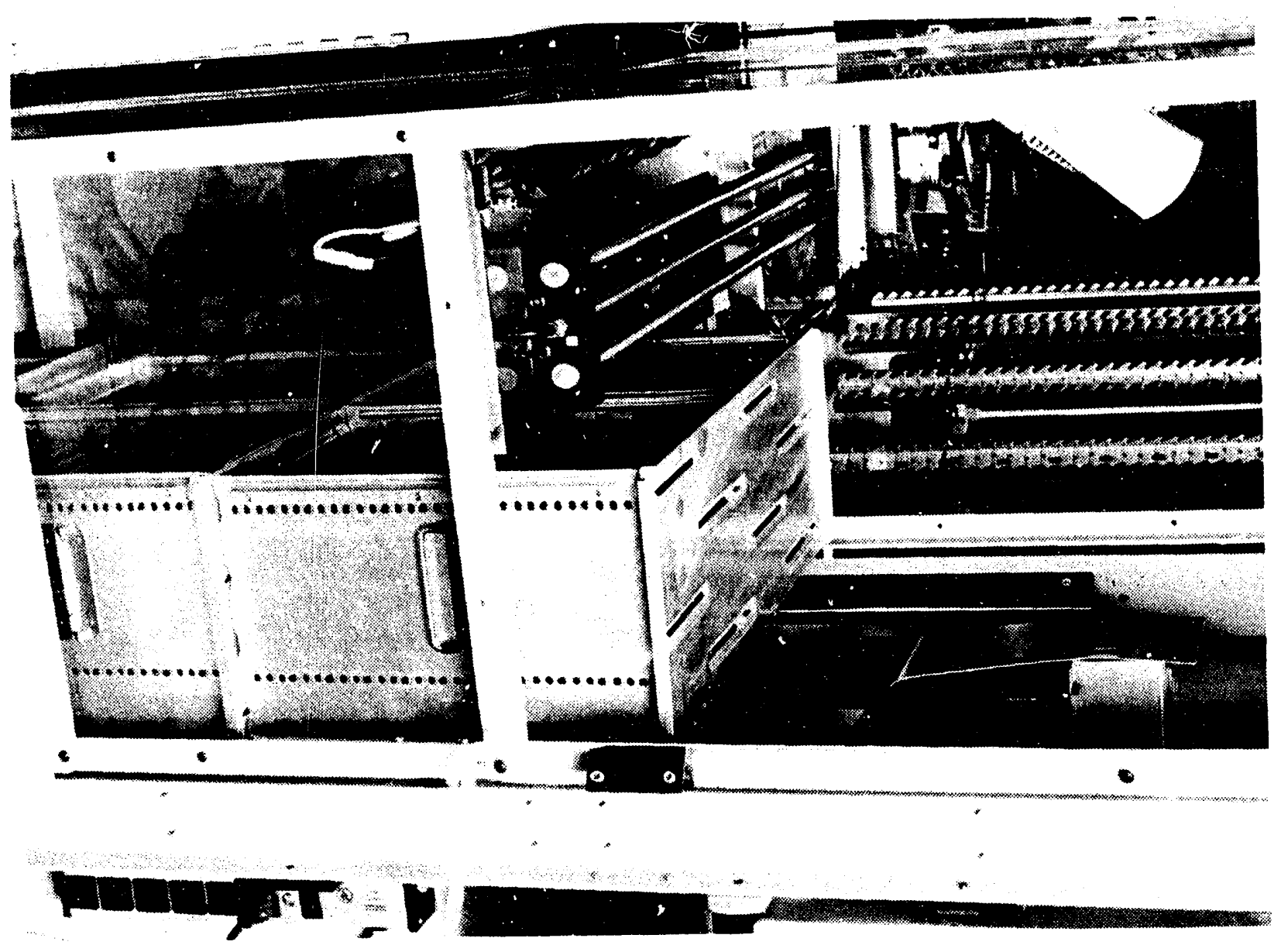

Figure 10. Tray Handling Mechanism 


\title{
REFERENCES
}

\begin{abstract}
${ }^{1} \mathrm{~K}$. J. Groot, Increased Robotic Capabilities for Lead Preparation and Component Insertion on Printed wiring Assemblies, (Topical Report). Kansas City Division: BDX-613-3806, September 1987 (Available from NTIS).
\end{abstract}

${ }^{2} \mathrm{~K}$. J. Groot, Review of Robotic Component Insertion System for Printed Wiring Assemblies, (Topical Report). Kansas City Division: BDX-613-3512, July 1986 (Available from NTIS). 

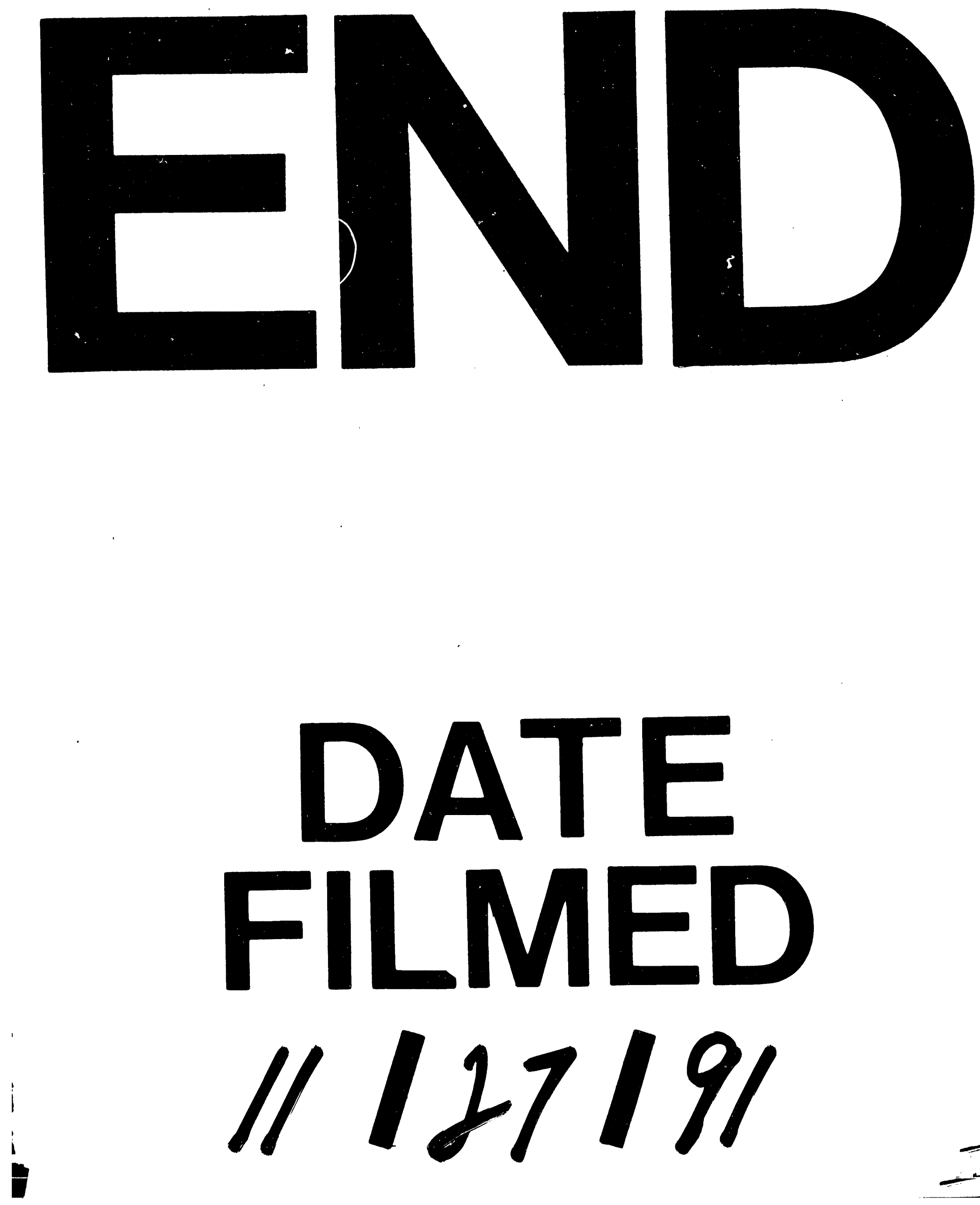
